\title{
Efeitos da associação de estresse físico e uso crônico de cloridrato de fluoxetina no córtex cerebral de ratos
}

\author{
Effects of the association of physical stress and chronic use of fluoxetine hydrochloride on the \\ cerebral cortex
}

Efectos de la asociación de estrés físico y el uso crónico de clorhidrato de fluoxetina en el cortex cerebral de ratas

Karinne da Costa Souza

ORCID: https://orcid.org/0000-0002-5139-7445 Universidade Federal de Alfenas, Brasil E-mail: karinne_souza24@hotmail.com

Micaella Rodrigues Guimarães Leite

ORCID: https://orcid.org/0000-0001-7779-0395 Universidade Federal de Alfenas, Brasil E-mail:rodriguesmicaella99@gmail.com

Alessandra Esteves

ORCID: https://orcid.org/0000-0002-0228-2940 Universidade Federal de Alfenas, Brasil E-mail:aesteves015@gmail.com

\begin{abstract}
Resumo
A exposição a fatores estressantes tem papel importante no desenvolvimento de transtornos depressivos. Os efeitos deletérios do estresse na neuroplasticidade e a apoptose podem ser acentuados pelo uso crônico de antidepressivos. Este estudo tem como objetivo avaliar e correlacionar os efeitos e as consequências do estresse físico (EF) associado ao tratamento de cloridrato de fluoxetina $(\mathrm{CF})$ no volume cerebral e espessura cortical de três áreas cerebrais. Foram utilizados cérebros de 25 ratos (Rattus norvegicus) machos da linhagem Wistar. Os animais foram divididos em 5 grupos: G1 - grupo controle; G2 - CF e sem EF; G3 - EF porém sem CF; G4 - CF até o dia anterior ao procedimento cirúrgico (EF) e G5 - CF até 30 dias após o procedimento cirúrgico (EF). Os resultados obtidos mostram que os grupos G3, G4 e G5 tiveram uma redução significativa no volume cerebral. Com relação a espessura cortical na área límbica os dados mostram que os grupos G4 e G5 tiveram uma redução significativa em relação ao G1, assim como os grupos G3, G4 e G5 também apresentaram uma diferença significativa em relação ao G2. Na área sensitiva os grupos G3, G4 e G5 tiveram uma redução expressiva quando comparados ao G1; o mesmo foi observado para G4 e G5 em relação aos grupos G2 e G3. Já em relação a área motora observou-se o mesmo para os grupos G3 e G4 em relação ao G1. Estes dados nos permitem concluir que, o estresse físico associado ou não ao uso crônico de cloridrato de fluoxetina diminui o volume cortical do cérebro de ratos machos como consequência desta redução de volume tem-se uma redução significativa da densidade cortical das áreas límbica, sensitiva e motora.
\end{abstract}

Palavras-chave: Córtex cerebral; Estresse físico; Cloridrato de fluoxetina. 


\begin{abstract}
Exposure to stressful factors plays an important role in development of depressive disorders. The deleterious effects of stress on neuroplasticity and apoptosis can be accentuated by chronic use of antidepressants. Thus, this study aims to evaluate and correlate the effects and consequences of physical stress (PS), associated with the treatment of fluoxetine hydrochloride (FC), on brain volume and cortical thickness of three brain areas. Brains of 25 male Wistar rats (Rattus norvegicus) were used. These animals were divided into 5 groups: G1 - control group; G2 - FC and without PS; G3 PS but without FC; G4 -FC until the day before surgical procedure (PS); and G5 - Animals treated with FC until 30 days after surgical procedure (PS). The results show that groups G3, G4 and G5 had a significant reduction in brain volume. Regarding cortical thickness in limbic area, the data show that groups G4 and G5 had a significant reduction compared to G1, as well as groups G3, G4 and G5 showed a significant difference compared to G2. In the sensitive area, groups G3, G4 and G5 had a significant reduction when compared to G1; the same was observed for G4 and G5 in relation to groups G2 and G3. In the motor area, a similar result was observed in groups G3 and G4 in relation to G1. The data allow to conclude that physical stress, associated or not with chronic use of fluoxetine hydrochloride, decreases the cortical volume of male rats brain and, as a consequence of reduction, there is a significant loss of cortical density in limbic, sensory and motor areas.
\end{abstract}

Keywords: Cerebral cortex; Physical stress; Fluoxetine hydrochloride.

\title{
Resumen
}

La exposición a factores estresantes tiene papel importante en el desarrollo de trastornos depresivos. Los efectos deletéreos del estrés en la neuroplasticidad y la apoptosis pueden verse acentuados por el uso crónico de antidepresivos. Ante esto, este estudio tiene por objetivo evaluar y correlacionar los efectos y consecuencias del estrés físico (EF) asociado al tratamiento con clorhidrato de fluoxetina (CF) en el volumen cerebral y espesura cortical, de tres áreas cerebrales. Fueron utilizados cerebros de 25 ratas (Rattus novegicus) machos del linaje Wistar. Los animales, fueron divididos en 5 grupos: G1 - grupo control; G2 - CF y sin EF; G3 - EF, pero sin CF; G4 - CF, hasta el día anterior al procedimiento quirúrgico (EF); G5 - CF, hasta 30 días después del procedimiento quirúrgico (EF). Los resultados obtenidos apuntaron que los grupos G3, G4 y G5 sufrieron reducción significativa en el volumen cerebral. En relación a la espesura cortical en el área límbica, los datos muestran que los grupos G4 y G5 tuvieron una reducción significativa en relación al G1, así como los grupos G3, G4 y G5, también presentaron una diferencia significativa en relación al G2. En el área sensitiva: los grupos G3, G4 y G5, tuvieron una reducción expresiva, cuando comparados al G1; lo mismo se observó para G4 y G5 en relación a los grupos G2 y G3. En el área motora, lo mismo se observó en los grupos G3 y G4 en relación al G1. Así, estos datos nos permiten concluir que: el estrés físico, así como el estrés físico asociado al uso crónico de clorhidrato de fluoxetina disminuye el volumen cortical del cerebro de machos de rata. A consecuencia de esta reducción de volumen, ocurre una reducción significativa de la densidad cortical de las áreas límbica, sensitiva y motora.

Palabras clave: Corteza cerebral; Estrés físico; Clorhidrato de fluoxetin.

\section{Introdução}

A depressão é uma patologia com condição frequente na atualidade devido ao estresse vivenciado no cotidiano da sociedade, consiste em uma associação de nível alto de incapacitação funcional, os principais fármacos utilizados para seu tratamento são os inibidores seletivos da recaptação de serotonina (IRSR), devida a sua grande procura por profissionais por apresentarem um menor efeito negativo nos indivíduos em fase de tratamento e por ser usado em um espaço de tempo menor garantindo um resultado eficiente. Dos fármacos usados a fluoxetina tem um grande destaque (Fleck et al., 2009).

A origem de patologias que acometem o sistema nervoso segundo alguns estudos tem sua origem através de estresses vivenciados no cotidiano, sendo esse estresse responsável por coordenar a resposta ao estresse quando um extensor excede um limite padrão, gerando assim devida a desordem gerada, uma disfunção cognitiva e comportamental de um indivíduo (Chrouso \& Gold, 1992). Embora haja uma pré disposição genética para estas fisiopatologias, mas ao incerto, está relacionada ao sistema da serotonina (5-HT), na qual a neurotrasmissão esteja irregular (Firk \& Markus, 2006).

O cloridrato de fluoxetina, um fármaco que atua na inibição e receptação de serotonina usado como antidepressivo por possuir uma vasta característica de atuação no tratamento de transtornos adversos, em princípio, possui uma grande eficácia por apresentar meia vida na sua eliminação em organismos em um período muito menor em comparação a outros inibidores seletivos da receptação de serotonina (IRSN's), mas em comparação a eficácia, a fluoxetina não é tão atuante como os 
antidepressivos tricíclicose outros usados para pacientes deprimidos (Benfiel, Heel \& Lewis, 1986; Feighner, 1999; Gingrich \& Hen, 2001; Silva \& De, 2008).

Desta forma, este estudo tem como objetivo avaliar e correlacionar os efeitos e as consequências do estresse físico associado ao tratamento de cloridrato de fluoxetina no volume cerebral e espessura cortical de três áreas cerebrais (motora, límbica e sensitiva).

\section{Metodologia}

O material utilizado neste projeto já faz parte do acervo de amostras do Departamento de Anatomia (DAnat) ICBUnifal-MG estando de acordo com os princípios éticos de utilização animal tendo sido aprovado pela Comissão de Ética em Experimentação Animal da Unifal-MG, protocolo nº 26/2019.

Desta forma, foram utilizados 25 cérebros de ratos (Rattus norvegicus) machos da linhagem Wistar, provenientes do Biotério da Universidade Federal de Alfenas UNIFAL- MG, os quais foram alojados em caixas contendo três animais cada uma, tratados com ração comercial e água à vontade e mantidos em ciclo de 12 horas claro-escuro.

\subsection{Grupos experimentais}

Os animais foram divididos em cinco (5) grupos experimentais ( $\mathrm{n}=5)$ sendo:

G1 - grupo controle; sem medicação (fluoxetina) e sem estresse físico (procedimento cirúrgico);

G2 - Animais tratados com cloridrato de fluoxetina e sem estresse físico;

G3 - Animais submetidos à estresse físico, porém sem fluoxetina;

G4 - Animais tratados com cloridrato de fluoxetina até o dia anterior ao procedimento cirúrgico;

G5 - Animais tratados com cloridrato de fluoxetina até 30 dias após o procedimento cirúrgico.

\subsection{Tratamento}

O tratamento consistiu na administração de $10 \mathrm{mg} / \mathrm{kg} / \mathrm{dia}$ de cloridrato de fluoxetina (Prozac®), o qual foi diluído em solução fisiológica estéril e administrada via intra-peritoneal (Cuquetto et al., 2012; Fernandes et al., 2012).

Os animais que não receberam o cloridrato de fluoxetina também se submeteram à injeção intra-peritoneal de solução fisiológica estéril, para mimetizar o estresse da aplicação e do próprio manejo com animal. O início da administração das substâncias foi de sessenta dias antes do procedimento cirúrgico, para que se caracterize o uso crônico de cloridrato de fluoxetina. Para os animais do grupo IV, no dia que antecedeu a cirurgia, se encerrou a administração da medicação. No grupo $\mathrm{V}$, os animais receberam a fluoxetina até o dia que antecedeu a eutanásia.

\subsection{Eutanásia dos animais e coleta das amostras}

Os animais foram eutanasiados por meio do anestésico Alotano®, e após a identificação de cada animal, iniciamos, o seguinte procedimento: após craniotomia os encéfalos foram inteiramente retirados e identificados, posteriormente lavados em solução fisiológica e fixados em paraformaldeído $4 \%$ em tampão fosfato pH 7,4 0,1M.

Os fragmentos foram processados seguindo-se a sequência padronizada nos procedimentos histológicos convencionais: desidratação em álcool, diafanização em xilol e inclusão em parafina. Cada amostra foi cortada em micrótomo Yidi® com espessura de $7 \mu \mathrm{m}$ e coradas com violeta cresil. De cada encéfalo foram retiradas amostras em cortes frontais, seriados e homotípicos para análise da espessura cortical então estabelecidas para este estudo. 


\subsection{Estimativa da espessura cortical do cérebro}

Para obtenção dos dados morfométricos da espessura cortical do cérebro, adaptamos a metodologia utilizada por Rowley et al., 2015; Dahnke et al., 2012; Scott e Thacker, 2005; Fischl e Dale, 2000. Para este estudo foram selecionados cinco (5) cortes de todos os cérebros; destes cinco cortes foram selecionados para análise aleatória. Foram mensuradas 6 áreas do córtex cerebral sendo 3 áreas de cada hemisfério cerebral (figura 1). A mensuração foi realizada ao microscópio de luz com aumento de 2.5x. Para a análise foi utilizado o software Axiovision Rel. 4.8.2 e Axiovision 4 Module InteractiveMensurement da marca Carl Zeiss.

Figura 1. Fotomicroscopia de cortes frontais de cérebros de rato evidenciando algumas das áreas do córtex cerebral que serão mensuradas (traço preto). Sendo CC: córtex cerebral, ec: cápsula externa, CPu: striatum, ic: cápsula interna, cg: cíngulo e fimbrias do hipocampo. Coloração violeta cresil. Aumento de 2.5x. Fonte: DAnat, ICB, Unifal-MG.

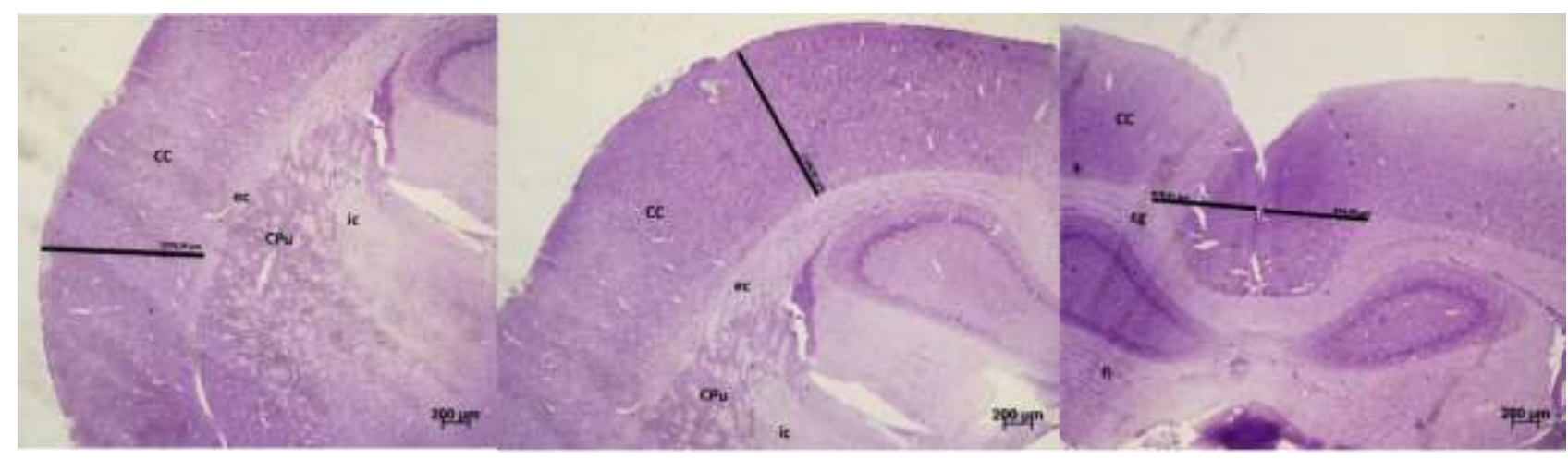

Fonte: Autores.

\subsection{Análise estatística}

O estudo foi conduzido em delineamento inteiramente casualizado. A análise estatística foi realizada por meio de Análise de Variância (One-Way ANOVA), seguida do teste de comparações de médias de Tukey. Utilizamos o software GraphPadPrism 8 (GraphPad Software, La Jolla, CA, EUA) e foi adotado o nível de significância de 5\% para todas as análises.

\section{Resultados}

Conforme demonstra o gráfico abaixo podemos observar que não houve diferenças significativas do G1 (grupo controle) em relação ao grupo G2, ou seja, o uso isolado de cloridrato de fluoxetina, porém sem o fator estresse físico envolvido não reduziu o volume cerebral dos animais destes grupos (Figura 2).

Já os dados obtidos do G1 diferem de forma significativa em relação aos grupos G3 e G4 ( $p<0,01$ ); animais que foram submetidos ao estresse físico e o uso do fármaco, até um dia anterior ao procedimento cirúrgico, respectivamente levam a uma redução significativa do volume cerebral, o que demonstra que só o estresse físico já é um fator predisponente e pode provocar danos consideráveis no cérebro.

Porém, os dados mais significativos foram observados entre os grupos G1 em relação ao grupo G5 (p<0,001), ou seja, animais tratados com cloridrato de fluoxetina até 30 dias após o procedimento cirúrgico (estresse físico) promoveu uma redução significativamente do volume cortical do cérebro. 
Figura 2. O gráfico demonstra a diferença significativa do volume cerebral observado entre os grupos controle (G1) em relação aos demais grupos experimentais. Sendo * $(\mathrm{p}<0,01) \mathrm{e} * *(\mathrm{P}<0,001)$.

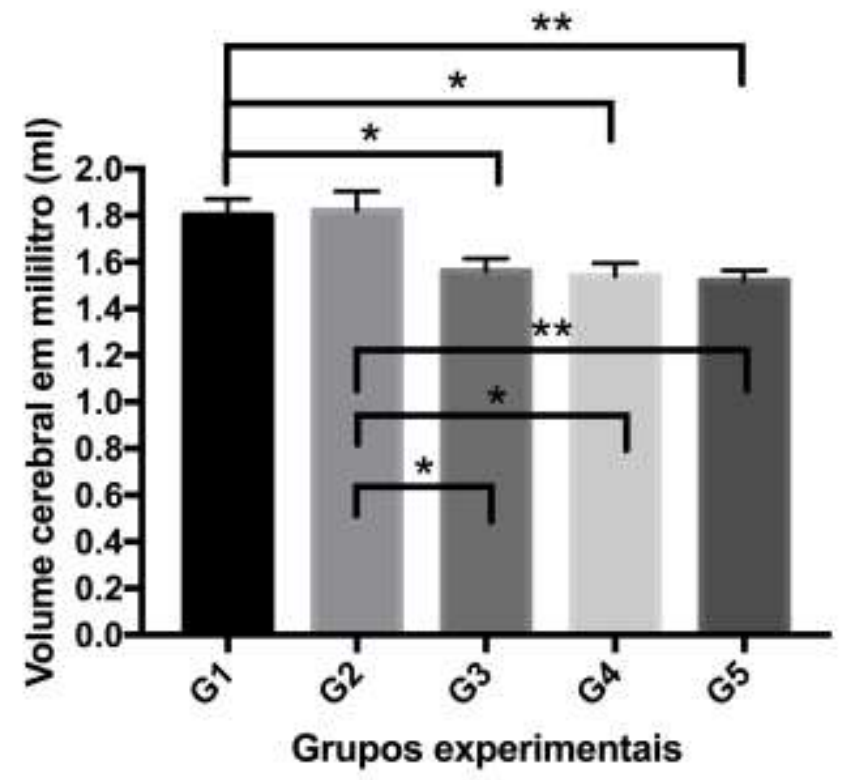

Fonte: Autores.

As mensurações obtidas em relação a densidade do córtex cerebral dizem respeito a espessura cortical de três áreas cerebrais: límbica, motora e sensitiva.

Ao observar os dados obtidos para cada grupo estudado, onde a área de interesse cortical foi a área límbica, o gráfico (Figura 3) mostra que o grupo de controle (G1) comparado com os grupos G2, G3 assim como os grupos G4 x G5 não se mostraram ser significativamente diferentes. Já quando comparado o grupo G1 com os grupos G4 e G5 os dados foram altamente significativo $(\mathrm{p}<0,0001)$.

Quando comparado o G2 como os demais grupos o mesmo se mostrou ser estatisticamente diferentes do G3 (p<0,01) e altamente significativo do G4 e G5 (p<0,0001). Quando se analisou comparativamente o grupo G3 em relação ao G4 e G5 podemos observar uma diferença estatística significativa $(\mathrm{p}<0,05)$. 
Figura 3. O gráfico demonstra a diferença significativa da espessura cortical da área límbica observado entre os grupos controle (G1) em relação aos demais grupos experimentais. Sendo * e ** $(\mathrm{p}<0,05)$ e $* * *(\mathrm{P}<0,01)$ e $* * * *(p<0,0001)$.

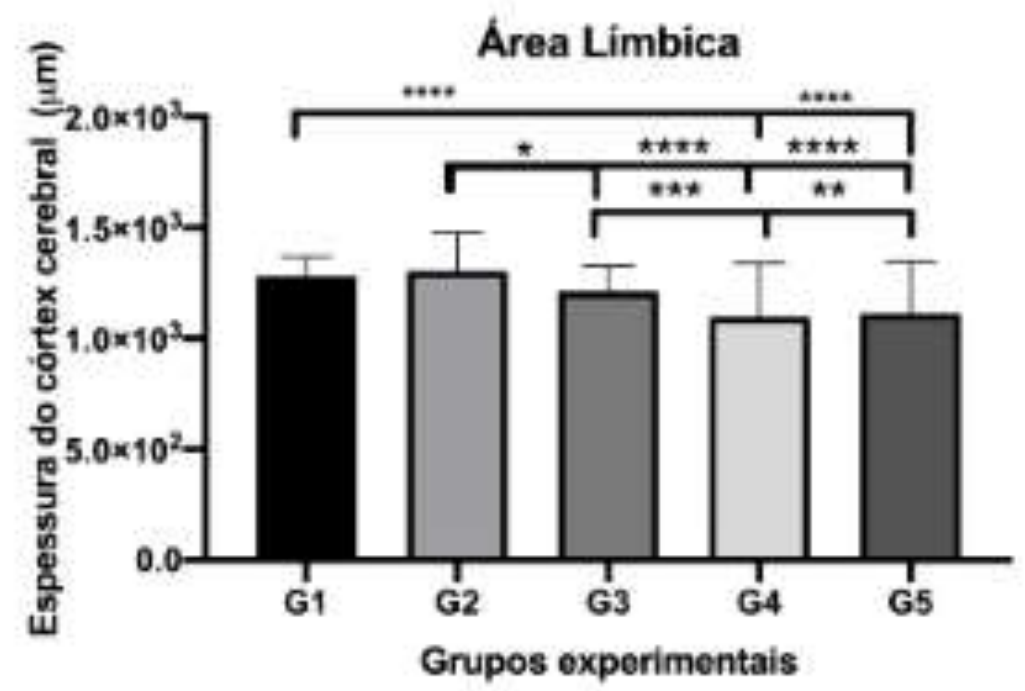

Fonte: Autores.

Em relação a análise estatística da área motora (Figura 4), obteve-se os seguintes dados: que o grupo de controle (G1) comparado com o grupo G2; G2 comparado ao G3 bem como o G4 em relação ao G5 não se mostraram ser diferentes significativamente.

Já quando comparado o grupo G1 com o grupo G3 que mostrou ser diferentes entre si $(\mathrm{p}<0,005)$ assim como se mostrou ser altamente significativo entre os G4 e G5 (p<0,0001).

Quando comparado o G2 como os grupos G4 e G5 mostrando-se ser altamente estatisticamente diferentes (p<0,0001). Quando analisado comparativamente o grupo G3 em relação ao G4 e G5 podemos observar uma diferença estatística significativa $(\mathrm{p}<0,005)$.

Figura 4. O gráfico demonstra a diferença significativa da espessura cortical da área sensitiva observado entre os grupos controle (G1) em relação aos demais grupos experimentais. Sendo ** e \#\# (p<0,005) e **** e \#\#\#\# (p<0,0001).

\section{Area Sensitiva}

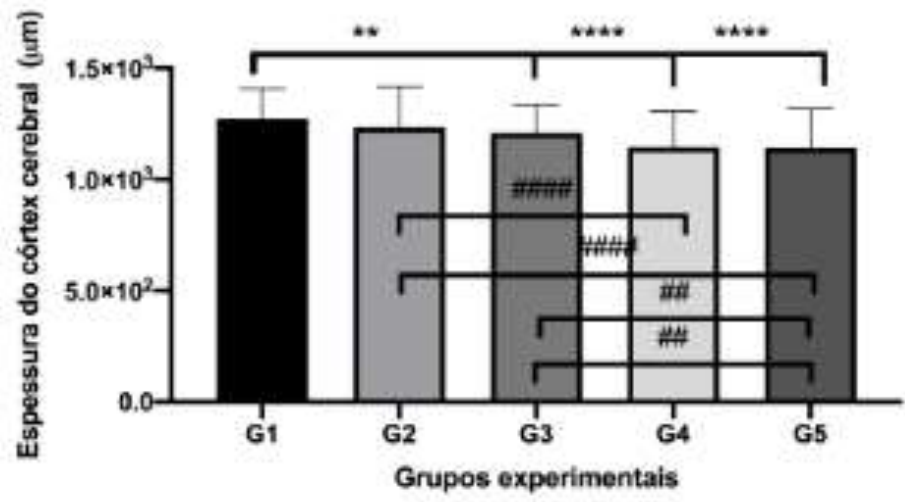

Fonte: Autores. 
Ao observar os dados obtidos da área motora, o gráfico (Figura 5) mostra que o grupo de controle (G1) comparado com os grupos G2 e G5 assim como o G2 com os demais grupos experimentais não se mostraram ser significativamente diferentes. Já quando comparado o grupo G1 com o grupo G3 e G4 os dados foram significativamente diferentes ( $<0,05)$.

Figura 5. O gráfico demonstra a diferença significativa da espessura cortical da área motora observado entre os grupos controle (G1) em relação aos grupos G3 e G4. Sendo * e ** ( $p<0,05)$.

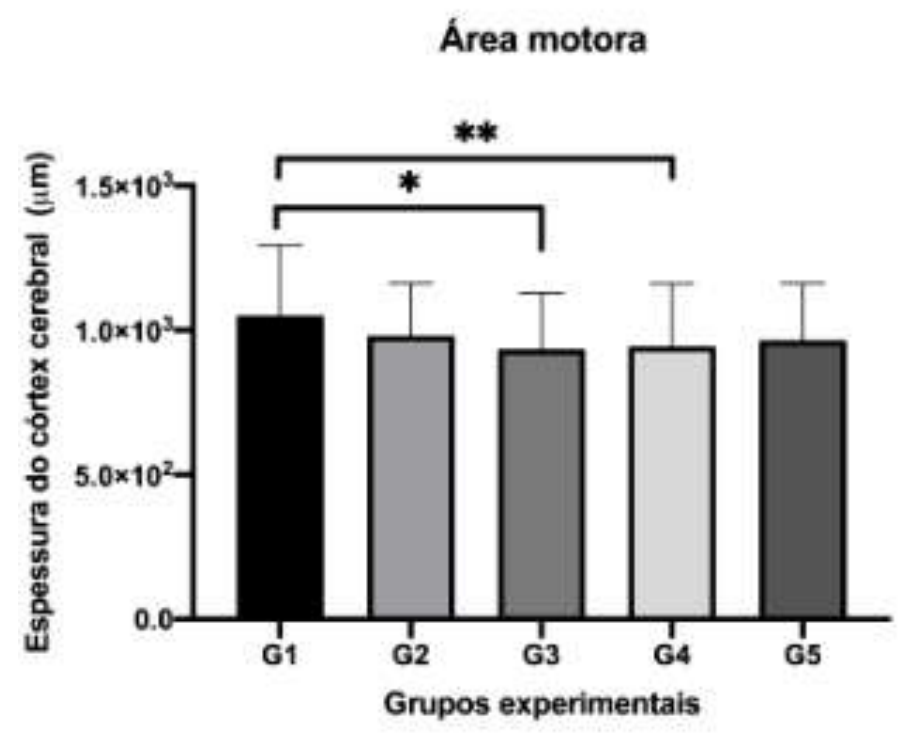

Fonte: Autores.

\section{Discussão}

Veena, Rao e Srikumar, 2011 relatam a importância de novos estudos relacionando a suposta neurogênese envolvida em alguns protocolos terapêuticos com antidepressivo, já que dados mostram que a regulação do estresse por meio de fármacos acaba por inibir a regulação correta da neurogênese levando inclusive ao acometimento da plasticidade celular do sistema nervoso central (SNC). Este recente trabalho vem em encontro com as afirmações de Veena, Rao e Srikumar, 2011 mostrando que o uso crônico o antidepressivo, cloridrato de fluoxetina pode, desencadear perda de volume cortical nas áreas sensitiva, motora e límbica, levando a prejuízos somestésicos, comportamentais e físicos.

A forma a qual cada organismo responde há administração de medicamentos sofre uma variação de um indivíduo para o outro, e isso segundo alguns estudos, tem relação direta com a variabilidade genética que é o principal fator de efeitos benéficos de um determinado fármaco em um individuo ou até mesmo gerar respostas contrárias em outros e isto não foge dos fármacos inibidores seletivos da receptação de serotonina (IRSR's) (White, Walline \& Barker, 2005). Diante disso, é possível sugerir que o uso crônico de cloridrato de fluoxetina associado ou não ao estresse físico desencadeou uma cascata de eventos negativos nas células nervosas e gliais no cérebro dos animais em estudo, levando a estas respostas contrárias ao esperado conforme relata White, Walline e Barker, 2005.

Czéh et al., 2007 relata que o uso crônico da fluoxetina pode alterar a proliferação celular quando submetido o indivíduo ao estresse crônico, afetando não somente células neuronais, como as células defesas do SNC, podendo associar á diminuição da espessura cortical devido ao acometimento das células de defesa não havendo a neurogenese das mesmas. Assim sendo, os achados deste trabalho corroboram com os dados obtidos por Czéh et al., 2007 que mostrou uma redução expressiva da espessura cortical com o uso crônico de cloridrato de fluoxetina. 
Hwang (2020) e Djordjevic (2012) relatam que o tratamento crônico de fluoxetina pode trazer a morte neuronal por apoptose por meio da oxidação por apresentar cobre e demais íons metálicos e alcalinos presentes, dados estes que reafirmam os dados apresentados neste recente trabalho.

$\mathrm{O}$ estresse aplicado a animais geralmente causa efeitos em áreas cerebrais tais como hipocampo e córtex pré-frontal (Bloss et al., 2010; Gárate et al., 2013; McAlliste et al., 2015) e uma das alternativas para o tratamento dessas patologias relacionadas ao estresse físico é o uso crônico de antidepressivos, onde estes fármacos tendem a reduzir a apoptose no hipocampo e aumentar a neurogênese na região afetada. Porém estudos realizados por Bremner, 2006 e Sousa et al., 2000 mostraram em imagens de ressonância magnética que, animais tratados com antidepressivos tiveram uma redução de volume do hipocampo achados estes que, podem ter relação com os dados obtidos neste trabalho tendo em vista que o hipocampo é uma área que mantem conexões nervosas diretas com as áreas límbica e sensitiva e se, houve uma redução expressiva do volume cortical destas áreas é possível que isso tenha refletido também no hipocampo.

Chrousos e Gold, 1992 relata que o uso de cloridrato de fluoxetina sobre transtornos generalizados bem como, sua eficácia nos variados tipos de estresse tem suas origens no sistema autônomo, porém o uso crônico pode desencadear uma alta elevação na resposta levando a alterações tanto comportamentais como também físicas. Este estudo pode estar de certa forma relacionado ao presente trabalho, pois as mudanças comportamentais e físicas descritas pelos autores podem, estar envolvidas na provável morte celular e consequente diminuição de volume nas áreas motora e límbica.

Estudos mostram a ação dos fármacos antidepressivos como o cloridrato de fluoxetina na neurogênese hipocampal, como de outras regiões do encéfalo, porém o uso crônico e a pausa no tratamento podem impedir a resposta de ação de fármacos. Testes feitos em camundongos insensíveis ao receptor da serotonina mostra que a neurogênese é bloqueada quando ocorre retomada ao tratamento ou mesmo quando o uso deste fármaco se faz por um período prolongado (Santarelli et al., 2003; Oquendo et al., 2013; Bosman et al., 2018). Os dados apresentados pelos autores podem ter relação com as áreas límbica e sensitiva encontradas neste recente trabalho, pois estas áreas mantêm conexões nervosas diretas entre si assim sendo, o uso crônico do fármaco cloridrato de fluoxetina associado ou não ao estresse físico, pode ter desencadeado a morte neuronal nestas áreas interferindo inclusive na neurogênese hipocampal.

A partir da observação de algumas condições neuropsiquiátricas, os neurocientistas suspeitaram de uma influência mais direta da emoção no movimento. Na depressão, por exemplo, a redução do movimento físico é um sintoma típico, quase sempre relacionado ao processamento emocional corrompido e à motivação reduzida. Especificamente, há evidências de que as informações límbicas afetam os circuitos motores provavelmente pela modulação da conexão entre o núcleo estriado ventral (núcleo da base) e o córtex motor, através da via nigro-talâmica (Aoki et al., 2019; Beatty \& Janelle, 2020). Em uma outra pesquisa, demonstrou-se que as emoções são capazes de modular o feedback dos nossos músculos, o que implica em consequências diretas para a nossa consciência corporal e prontidão para reagir a situações emocionais. No caso, tristeza fizeram com que esses fusos respondessem de maneira diferente ao movimento (Ackerley et al., 2017; Wulf \& Lewthwaite 2016). Diante dos achados obtidos neste recente trabalho e os dados obtidos pelos autores citados fica claro que, algumas doenças neuropsiquiátricas ou mesmo uso fármacos de forma crônica afetam diretamente os circuitos motores em consequência das interferências sobre o sistema límbico.

\section{Conclusão}

Estes dados nos permitem concluir que, o estresse físico isoladamente assim como o estresse físico associado ao uso crônico de cloridrato de fluoxetina diminui o volume cortical do cérebro de ratos machos como consequência desta redução de volume tem-se uma redução expressiva da densidade cortical das áreas límbica, sensitiva e motora levando a prejuízos somestésicos, comportamentais e físicos nos usuários destes fármacos. 
Pelo fato do Cloridrato de fluoxetina ser um fármaco frequentemente utilizado sugere-se que, outras áreas corticais e subcorticais sejam estudadas para analisar os possíveis efeitos deletérios também nestas áreas.

\section{Referências}

Ackerley, R., Aimonetti, J. M., \& Ribot-Ciscar, E. (2017). Emotions alter muscle proprioceptive coding of movements in humans. Scientific Reports, 7(1), 19. https://doi.org/10.1038/s41598-017-08721-4

Aoki, S., Smith, J. B., Li, H., Yan, X., Igarashi, M., Coulon, P., Wickens, J. R., Ruigrok, T. J., \& Jin, X. (2019). An open cortico-basal ganglia loop allows limbic control over motor output via the nigrothalamic pathway. ELife, 8. https://doi.org/10.7554/eLife.49995

Benfield, P., Heel, R. C., \& Lewis, S. P. (1986). Fluoxetine. Drugs, 32(6), 481-508. https://doi.org/10.2165/00003495-198632060-00002

Bloss, E. B., Janssen, W. G., McEwen, B. S., \& Morrison, J. H. (2010). Interactive effects of stress and aging on structural plasticity in the prefrontal cortex. Journal of Neuroscience, 30(19), 6726-6731. https://doi.org/10.1523/JNEUROSCI.0759-10.2010

Bosman, R. C., Waumans, R. C., Jacobs, G. E., Oude Voshaar, R. C., Muntingh, A. D. T., Batelaan, N. M., \& van Balkom, A. J. L. M. (2018). Failure to Respond after Reinstatement of Antidepressant Medication: A Systematic Review. Psychotherapy and Psychosomatics, 87(5), 268-275. https://doi.org/10.1159/000491550

Bremner, J. D. (2006). Stress and brain atrophy. CNS \& Neurological Disorders Drug Targets, 5(5), 503-512. http://www.ncbi.nlm.nih.gov/pubmed/17073653\%5Cnhttp://www.pubmedcentral.nih.gov/articlerender.fcgi?artid=PMC3269810

Chrousos, G. P., \& Gold, P. W. (1992). The Concepts of Stress and Stress System Disorders: Overview of Physical and Behavioral Homeostasis. JAMA, 267(9), 1244-1252. https://doi.org/10.1001/jama.1992.03480090092034

Czéh, B., Müller-Keuker, J. I. H., Rygula, R., Abumaria, N., Hiemke, C., Domenici, E., \& Fuchs, E. (2007). Chronic social stress inhibits cell proliferation in the adult medial prefrontal cortex: Hemispheric asymmetry and reversal by fluoxetine treatment. Neuropsychopharmacology, 32(7), 1490-1503. https://doi.org/10.1038/sj.npp.1301275

Djordjevic, A., Djordjevic, J., Elaković, I., Adzic, M., Matić, G., \& Radojcic, M. B. (2012). Effects of fluoxetine on plasticity and apoptosis evoked by chronic stress in rat prefrontal cortex. European Journal of Pharmacology, 693(1-3), 37-44. https://doi.org/10.1016/j.ejphar.2012.07.042

Feighner, J. P. (1999). Mechanism of action of antidepressant medications. The Journal of Clinical Psychiatry, 60 Suppl 4, 3-4.

Firk, C., \& Markus, C. R. (2006). Review: Serotonin by stress interaction: a susceptibility factor for the development of depression? Journal of Psychopharmacology, 21(5), 538-544. https://doi.org/10.1177/0269881106075588

Fleck, M. P., Berlim, M. T., Lafer, B., Sougey, E. B., Del Porto, J. A., Brasil, M. A., Juruena, M. F., \& Hetem, L. A. (2009). [Review of the guidelines of the Brazilian Medical Association for the treatment of depression (Complete version)]. Revista Brasileira de Psiquiatria (Sao Paulo, Brazil : 1999), 31 Suppl 1, S7-17. https://doi.org/10.1590/S1516-44462009000500003

Gárate, I., Garcia-Bueno, B., Madrigal, J. L. M., Caso, J. R., Alou, L., Gomez-Lus, M. L., Micó, J. A., \& Leza, J. C. (2013). Stress-induced neuroinflammation: Role of the toll-like receptor-4 pathway. Biological Psychiatry, 73(1), 32-43. https://doi.org/10.1016/j.biopsych.2012.07.005

Gingrich, J. A., \& Hen, R. (2001). Dissecting the role of the serotonin system in neuropsychiatric disorders using knockout mice. Psychopharmacology, 155(1), 1-10. https://doi.org/10.1007/s002130000573

Hwang, S., \& Kim, J.-K. (2020). Fluoxetine Induces Apoptotic and Oxidative Neuronal Death Associated with The Influx of Copper Ions in Cultured Neuronal Cells. Chonnam Medical Journal, 56(1), 20. https://doi.org/10.4068/cmj.2020.56.1.20

McAllister, B. B., Spanswick, S. C., Patel, P. P., Barneto, A. A., \& Dyck, R. H. (2015). The effects of chronic fluoxetine treatment following injury of medial frontal cortex in mice. Behavioural Brain Research, 290, 102-116. https://doi.org/10.1016/j.bbr.2015.04.049

Oquendo, M. A., Echavarria, G., Galfalvy, H. C., Grunebaum, M. F., Burke, A., Barrera, A., Cooper, T. B., Malone, K. M., \& Mann, J. J. (2003). Lower cortisol levels in depressed patients with comorbid post-traumatic stress disorder. Neuropsychopharmacology, 28(3), 591-598. https://doi.org/10.1038/sj.npp.1300050

Santarelli, L., Saxe, M., Gross, C., Surget, A., Battaglia, F., Dulawa, S., Weisstaub, N., Lee, J., Duman, R., Arancio, O., Belzung, C., \& Hen, R. (2003). Requirement of hippocampal neurogenesis for the behavioral effects of antidepressants. Science (New York, N.Y.), 301(5634), 805-809. https://doi.org/10.1126/science. 1083328

Silva, D. K., \& De, F. M. (2008). Pharmacogenetics of selective serotonine reuptake inhibitors: A review [Portuguese] Farmacogenetica de inibidores seletivos de recaptacao de serotonina: Uma revisao. Revista de Psiquiatria Do Rio Grande Do Sul, 30(1 SUPPL.).

Sousa, N., Lukoyanov, N. V, Madeira, M. D., Almeida, O. F., \& Paula-Barbosa, M. M. (2000). Reorganization of the morphology of hippocampal neurites and synapses after stress-induced damage correlates with behavioral improvement. Neuroscience, 97(2), 253-266. https://doi.org/10.1016/s0306-4522(00)00050-6

Veena, J., Rao, B. S. S., \& Srikumar, B. N. (2011). Regulation of adult neurogenesis in the hippocampus by stress, acetylcholine and dopamine. Journal of Natural Science, Biology, and Medicine, 2(1), 26-37. https://doi.org/10.4103/0976-9668.82312

White, K. J., Walline, C. C., \& Barker, E. L. (2005). Serotonin transporters: implications for antidepressant drug development. The AAPS Journal, 7(2), E42133. https://doi.org/10.1208/aapsj070242

Wulf, G., \& Lewthwaite, R. (2016). Optimizing performance through intrinsic motivation and attention for learning: The OPTIMAL theory of motor learning. Psychonomic Bulletin and Review, 23(5), 1382-1414. https://doi.org/10.3758/s13423-015-0999-9 